\title{
Analysis of key technologies in the Internet of things
}

\author{
Wang Rui ${ }^{1,}$ a , Wang Jinguo ${ }^{2, b^{*}}$ (corresponding author), Wang $\mathrm{Na}^{3, \mathrm{c}}$ \\ ${ }^{1}$ Department of Information Engineering,Jilin Business and Technology College \\ China \\ ${ }^{2}$ Department of Urology, the First Hospital of Jilin University,China \\ ${ }^{3}$ Department of Anesthesiology, the First Hospital of Jilin University, China

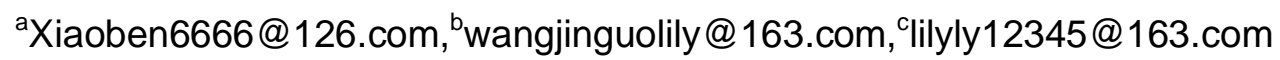

Keywords: Internet of things. RFID. ZigBee. Cloud computing.

Abstract. It is known as the third wave of the information industry after the personal computer and the Internet technology. IOT is an important breakthrough in the new century. On the basis of analyzing the system structure and technical characteristics of the Internet of things, the key technology of the Internet of things is deeply studied. The radio frequency identification technology(RFID), sensor technology, network communication technology and cloud computing are analyzed in detail. Finally, it illustrates the wide application prospect of the Internet of things.

\section{Introduction}

In 1999, American Auto-ID first proposed the concept of "Internet of things" (IOT), which is mainly based on the item coding, radio frequency identification technology and Internet technology. In the Information Society World Summit held in Tunisia in 2005, the International Telecommunication Union released the standard of the Internet of things, and expanded the meaning of the concept. Report pointed out that the goal of information and communication technology is no longer any time, any place connect anyone, but further development to anyone, at any time, any place can be legitimate connection to any goods, and all the objects can exchange information through the network, so that all things connected and constitute of Internet of things. In order to achieve this goal, sensor technology, radio frequency identification technology, network technology and embedded technology potential will be further excavated and will be more widely used. IOT industry development prospects are very optimistic, the market potential is also very large[1].

Using the Internet of things technology, can improve the economic efficiency, reduce operating costs. In the near future, many items will be perceived in various fields. Some experts predict that the Internet of things is a new information industry peak after the computer, the Internet and mobile communications network and other industries[2].

\section{Architecture and key technologies of the Internet of things}

The architecture of the IOT. Usually the IOT is divided into three levels: perception layer, network layer, application layer. The main function of the perception layer is overall perception, it can acquisition information whenever and wherever by using RFID, sensors, two-dimensional code. RFID technology, sensing and control technology, short distance wireless communication technology is the main technology on the perception layer, including chip development, communication protocol research, materials, smart node power supply and other segments[3]. 
The main function of the network layer is to realize the two-way transmission between the perception data and control information, and through the integration of telecommunication networks and the Internet to transmit the information in real time. After the sensor senses the item information, need to transfer to the background through the network to deal with. At present, the advanced technology of network transmission information application includes[4]: IPV6, New type of wireless communication network (3G、4G、ZIGBEE and so on), Ad hoc network technology. It is developing faster transmission speed, wider bandwidth, higher spectrum utilization, more intelligent access and network management. Network layer also has the function of information storage, network management and so on.

The application layer is analysis and processing of the data, to provide users with a specific services. Cloud computing platform is the storage of mass data, analysis platform, is not only an important part of the Internet of things, but also the basis for application of many applications. Application layer is the goal of the development of things, the software development, intelligent control technology will provide users with a variety of application of the IOT.

\section{Key technologies in the IOT}

There are a lot of new technologies in the Internet of things, the key technology of which is radio frequency identification technology(RFID), sensor technology, network communication technology and cloud computing. Here are several major key technologies to do a brief introduction.

Working principle and system composition of RFID. RFID is the abbreviation of radio frequency identification, also calls electronic tags. RFID technology is an automatic identification technology which began in the 90's in twentieth Century, it uses radio frequency signal through space coupling to realize the non-contact information transmission, and achieves the purpose of identification through the information transmitted[].

Its principle: after the label enters the magnetic field, if it can receive the reader sending out the special radio frequency signal, the product information stored in the chip can be transmitted by the energy of the induced current. The RFID should at least include the following two parts, one is the reader, the other is the electronic tag, in addition, antenna, host, etc. According to the different application purposes and application environment, RFID system will different. But from the work principle of RFID system, the system is composed of signal transmitter, signal receiver and transmitter receiver.

One of the most important applications of the IOT is the RFID tag. By attached tag on the good, we construct the information network on the basis of the existing internet. RFID releases data collected from the cable's constraints, improves the speed and flexibility of data collected.

ZigBee technology. Wireless sensor network (WSN) is a multi-hop self-organizing network system formed by a large number of sensor nodes through wireless communication. Its purpose is cooperative sensing, collecting and processing network information of objects in the network coverage area. It can realize data collection and quantification, processing fusion and transmission application. It is another kind of data collection technology of the IOT. The standard of wireless sensor network is ZigBee. ZigBee is a wireless network protocol formulated by ZigBee Alliance. Is a two-way wireless access technology of close distance, low power consumption, low data rate, low complexity, low-cost, which is mainly suitable for automatic control and remote monitoring. ZigBee is a highly reliable wireless data transmission network, which is similar to CDMA and GSM networks. ZigBee data transmission module is similar to the mobile network base station. ZigBee technology constitutes the wireless data transmission network platform up to 65000 wireless data 
transmission module. In the whole the network, each ZigBee network data transmission module can communicate with each other, and the distance between each network node can be expanded from the standard $75 \mathrm{~m}$ to infinite. Unlike CDMA network or GSM network, ZigBee network is established mainly for industrial automation control data transmission, therefore, it must have the simple, easy to use, reliable work, low price characteristics. Each ZigBee network node, not only can be as monitoring objects, such as the sensor connected on it can directly supervisory and collection data, but also automatically converted to the data other network nodes pass on.

Because of this technique providing high connectivity support mesh network, and the data transfers automatic routing between devices. Therefore, it is very simple to install the zigBee device in the existing environment. ZigBee's low power requirements also means lower maintenance, which makes it very suitable for consumer applications. At present, ZigBee technology is suitable for many applications: from industrial electric light switch to electric light components, electrical appliances, home electronics products.

Cloud Computing. In the information age, it is very important to deal with the information effectively. In the Internet of things, there is a large scale, mass data need to be processed, so the data processing capacity of a higher demand. Cloud computing is the integration product of traditional computer technology and network technology, such as grid computing, distributed computing, parallel computing, utility computing, network storage, virtualization, load balancing, etc. Cloud computing is a style of computing in which dynamically scalable and often virtualized resources are provided as a service over the Internet. It aims to integrate many relatively low cost computing entities into a perfect system with powerful computing capabilities through the network. Cloud computing a core idea is through continuously improve the processing ability of the "cloud", and to reduce the processing burden on the terminal. The terminal is simplified into a simple input and output devices, and according to the need to enjoy the "cloud" of the powerful computing capabilities. Cloud computing applications include such a thought: combine strength with each, every member could use it.

Cloud computing provides the most reliable, the most secure data storage center, users do not have to worry about data loss, virus intrusion and other trouble. Cloud computing can easily achieve data sharing between different devices and applications. Cloud computing provides almost unlimited potential for the user to use the network. For data storage and management provides an almost infinite space, also provides almost infinitely powerful computing ability for all kinds of application. This is only use personal computers or mobile phone client application can not be realized.

\section{Strategy suggestion for the IOT development}

Key technologies need to break. From the technical level, the IOT is a multidisciplinary nature, this industry has a lot of technical problems to be resolved.

1.Sensing technology. Sensor is the basis of the IOT. Sensor chip in China, from technology to manufacturing process, are behind the developed countries. Such as sensor node power supply problem, is a technical difficulty in the application of wireless sensor network in the practical application.

2.RFID technology. At present, China's RFID lack of key core technology, especially in the ultra high frequency RFID.Ultra high frequency RFID technology is a high threshold, the domestic development is later, the technology is relatively short.

3. Cloud computing. How to ensure that the data privacy that store in cloud service is not illegal use, not only need to improve the technology, but also need to further improve the law. Some data is the 
business secrets, the security of data is related to the survival and development of enterprises. If the security is not solve, it will affect application of the cloud computing in the enterprise.

4.Lack of uniform standard system. Key technology to be a breakthrough, the development more scattered, the system structure has not been established, the above problems are the main reasons for the lack of the standard system. The development of the Internet of things is facing the following challenges: first, the technical standards. Technical standards is the premise of the IOT development. The other is the industry standard, industry standard is the short board of the IOT development.

\section{References}

[1]Fleisch E. What is the Internet of Things? An economic perspective. http://www.internet-of-things.org/cl-pdf/AUTOUIDLABS-WP-BIZAPP-53.PDF.2010

[2] Guy Pujolle, An Autonomic - oriented Architecture for the Internet of Things[J]. IEEE. 2006.

[3] Inge. Architecture for the Internet of Things(IoT): API and interconnect[J]. IEEE.2008.

[4] Huansheng NING. Layered Structure and Management in Internet of Things[J]. IEEE. 2008. 